\title{
Pedestrian forward projection after vehicle collision
}

\author{
Paulo A.G. Piloto*, Vitor M. E. Teixeira ${ }^{* *}$ \\ *Polytechnic Instituteof Bragança;Campus Santa Apolónia;Bragança;Portugal; ppiloto@ipb.pt \\ **Polytechnic Instituteof Bragança;Campus Santa Apolónia;Bragança;Portugal; vitormanuell@ sapo.pt
}

Special Issue- “Biomechanics”

\begin{abstract}
This paper is focused in the assessment of the relation between the vehicle collision velocity, the pedestrian throw distance and the pedestrian injury, after vehicle-pedestrian collision with forward projection. A few parameters were considered, in particular the collision velocity (8), the pedestrian mass and height (8), and the mass of the vehicle (3). The results of the throw distance projection were compared using two solution methods (simple and advanced), the results of other authors and with 15 real data accident with forward projections. A total of 192 different conditions were simulated, being the results in good agreement. The injury of the pedestrian was calculated, based on two injury criteria (GSI and FFC), aiming to define a simple relation between pedestrian injury and the vehicle collision velocity. DOI: https:/doi.org/10.24243/JMEB/2.5.161
\end{abstract}

$\underline{\text { Research Article }}$

\begin{tabular}{cc}
\multicolumn{2}{c}{ Article History } \\
Received & $05 / 11 / 2017$ \\
Revised & $15 / 11 / 2017$ \\
Accepted & $06 / 12 / 2017$
\end{tabular}

Recommended by Editors Elza M. Fonseca and Maria Goreti Fernandes

\section{Introduction}

The number of pedestrian overthrowing caused by the collision of vehicles depends on the location (inside and outside urban areas) and depends on the country where it occurs. In Portugal, over the last decade, there has been a significant reduction, in particular those that occurred outside urban areas [1]. According to the European road safety statistics (19 members) [2], Portugal ranks 13th in terms of number of fatalities per million inhabitants (14.8), while Sweden ranks 1 st with the lowest number (6.1). The percentage of fatalities due to road accidents in relation to the total number of victims in road accidents was $16.1 \%$ in 2006 . The authorities are usually faced with the need to analyse collisions between pedestrians and vehicles, particularly when human lives are involved. This paper deals with the analysis of forward projection. This type of accident is one of the categorized overthrowing cause by vehicles and is classified since 1981 [3], taking in to consideration a study of 460 collisions between pedestrians and vehicles in Northern California.

Forward projection trajectory occurs when the height above the road to the collision point of the vehicle is located above the centre of mass of the pedestrian. The pedestrian is projected forwards and in contact with the ground. This type of trajectory normally occurs with small height pedestrians when struck by cars and with any height of pedestrians when struck by flat fronted vehicles, see Fig 1. 


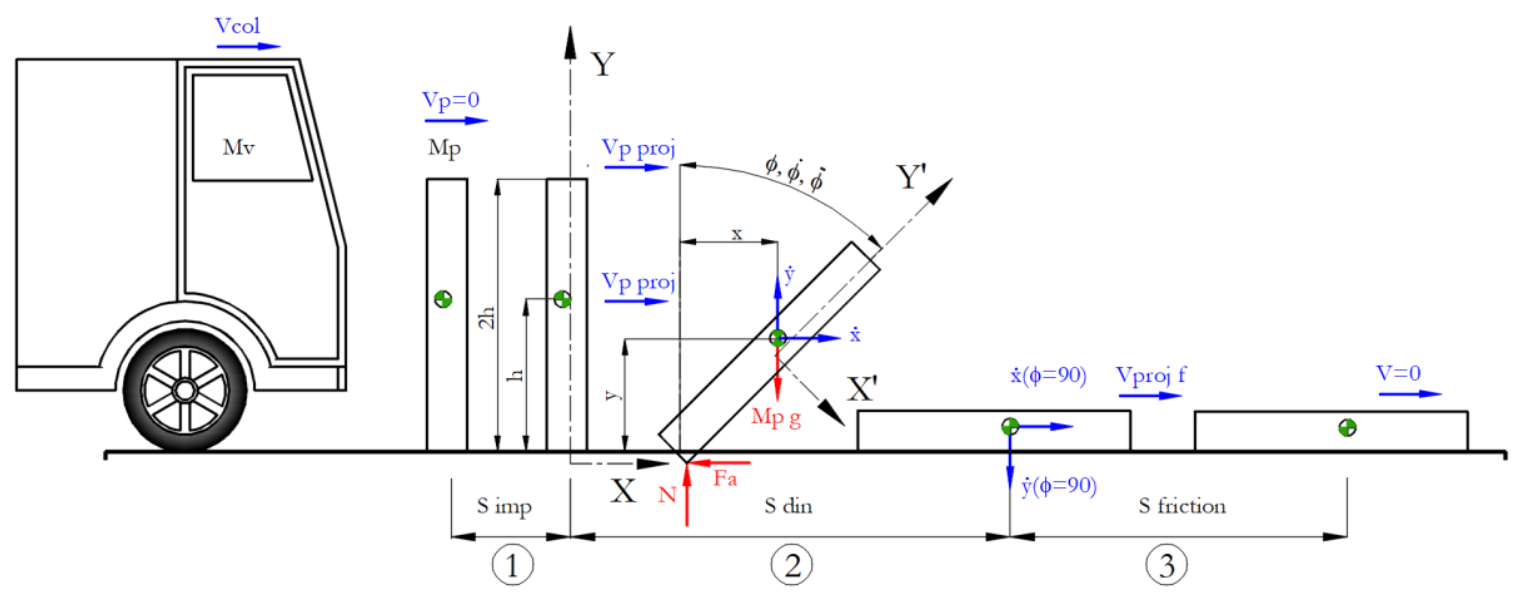

Fig.1 Pedestrian trajectory under frontal projection.

In the context of the research of road accidents, the collision velocity of the vehicle is the most important factor in the reconstruction of road accidents. The measurement of the pedestrian throw distance is decisive for the calculation of the collision velocity. There are several factors that influence the relationship between the pedestrian throw distance and the collision velocity, in addition to the collision mechanisms that can be observed in between the vehicle and the pedestrian and in between the pedestrian with the road. For this reason, 192 different conditions were analysed, resulting from 8 different collision velocities (Vcol1-Vcol8), 8 different pedestrians (P1-P8) and 3 different vehicles (Mv1-Mv3), see Table 1.

Table 1 Different parameters to analyse vehicle-pedestrian accident scenarios.

\begin{tabular}{|c|c|c|}
\hline $\mathrm{Mv}[\mathrm{kg}]$ & Vcol $[\mathrm{km} / \mathrm{h}]$ & $\mathrm{P}=\mathrm{Mp}[\mathrm{kg}] / 2 \mathrm{~h}[\mathrm{~m}]$ \\
\hline $\mathrm{Mv} 1=6500$ & $\mathrm{Vcol1}=20$ & $\mathrm{P} 1=52.40 / 1.450$ \\
\hline $\mathrm{Mv} 2=11500$ & $\mathrm{~V} \operatorname{col} 2=30$ & $\mathrm{P} 2=56.20 / 1.500$ \\
\hline \multirow[t]{6}{*}{ Mv3 $=17030$} & $V \operatorname{col} 3=40$ & $\mathrm{P} 3=60.00 / 1.550$ \\
\hline & $\mathrm{Vcol} 4=50$ & $\mathrm{P} 4=64.00 / 1.600$ \\
\hline & $V \operatorname{col} 5=60$ & $\mathrm{P} 5=68.20 / 1.650$ \\
\hline & $V \operatorname{col} 6=70$ & $P 6=72.40 / 1.700$ \\
\hline & $\mathrm{V} \operatorname{col} 7=80$ & $\mathrm{P} 7=76.80 / 1.750$ \\
\hline & $V \operatorname{col} 8=90$ & $\mathrm{P} 8=80.00 / 1.835$ \\
\hline
\end{tabular}

In the following sections, two solution methods are used to estimate pedestrian throw distance: the simplified method and the advanced calculation method. The simplified method derives from the 2D fundamental equations of the dynamics of rigid bodies, whereas the advanced calculation method derives from the application of the same fundamentals, but with the use of 3D articulated anatomical segments. Both results are compared with 15 results of real forward projection accidents investigated in UK [4], between 2001 and 2003.

This type pedestrian overthrowing can be subdivided by three phases, see Fig 1 . In the $1^{\text {st }}$ phase, the impact of the vehicle with the pedestrian occurs over a very short period of time [5], [6] and will cause the pedestrian to accelerate forwards. In the $2^{\text {nd }}$ phase, longer stage, the pedestrian goes into general plane motion, beginning to rotate down onto the road due to the friction force in their feet, with the body falling down, touching the ground and loosing horizontal velocity. In the $3^{\text {rd }}$ phase, the pedestrian is considered in contact with the road, being subjected to a friction action until final position. The summation of the all the horizontal displacements: " $S_{\text {imp }}$ " for the $1^{\text {st }}$ phase, " $S_{\operatorname{din}}$ " for the $2^{\text {nd }}$ phase and " $S_{\text {fricion }}$ " for the $3^{\text {rd }}$ phase, will provide the total pedestrian throw distance " $S_{\text {total }}$ "

The injury of the pedestrians can be predicted by the analysis of the head acceleration, normally used for GSI criterion (Gadd Severity Index) and predicted by the analysis of the joint force produced in the femur, normally used 
for FFC criterion (Femur Force Criterion). Injury occurs if the prediction of the biomechanical parameter is severed and beyond the specific limit.

\section{The simplified method}

The pedestrian throw distance " $S_{\text {total }}$ " was based on 2D dynamic analysis of a simplified rigid body, with rectangle shape. During the impact phase, the linear momentum is transferred from the vehicle of mass $M_{v}$ to the pedestrian of mass $M_{p}$, see Eq 1 .

$$
M_{v} v_{c o l}=M_{v} v_{v \text { proj }}+M_{p} v_{p \text { proj }}
$$

The relationship between the velocities of the two bodies before impact ( $v_{p}$ for pedestrian and $v_{c o l}$ for vehicle), and the velocities after impact ( $v_{p \text { proj }}$ and $v_{v p r o j}$ ), is characterized by the coefficient of restitution (e), see Eq (2).

$$
e=v_{v \text { proj }}-v_{\text {p proj }} / v_{p}-v_{c o l}
$$

The value of the coefficient of restitution varies with the value of the collision velocity, according to studies developed by Wood and Walsh [6] on the tests developed by Lucchini and Weissner [7], see Eq (3).

$$
e=\left\{\begin{array}{llll}
0.12-0.006 v_{c o l} & \text { if } & v_{c o l}<20 & {[\mathrm{~m} / \mathrm{s}]} \\
0.001 & \text { if } & v_{c o l} \geq 20 & {[\mathrm{~m} / \mathrm{s}]}
\end{array}\right.
$$

This $1^{\text {st }}$ phase is developed during the short period of time $\left(t_{i m p}\right)$, assuming a uniform rectilinear motion. For this motion, a mean contact time of 0.056 [s] was defined [5], which allows for the calculation of $S_{i m p}$. At the end of the impact phase, the transfer of linear momentum is assumed complete, and no interaction between the vehicle and the pedestrian is considered in the two subsequent phases.

During the general plane motion, $2^{\text {nd }}$ phase, the pedestrian acquires the initial velocity $v_{p p r o j}$, assuming that the pedestrian keeps the feet in contact with the ground. This contact implies the existence of a frictional force that will be responsible for this type of motion. The pedestrian will be subject to gravity load throughout this period, being the dynamic equilibrium expressed by Eqs (4)-(5). Eq (4) is normally written in the fixed Cartesian coordinate system XY, while Eq (5) is normally written in a moving coordinate system $X^{\prime} Y^{\prime}$, see Fig 1.

$$
\begin{aligned}
& \sum \vec{F}=m \times \vec{a}_{C M} \\
& \sum \vec{M}_{C M}=\dot{\vec{H}}_{C M}
\end{aligned}
$$

The pedestrian motion restriction insures that the pedestrian remains in contact with the ground using Eq (6). This equation allows the coupling between the vertical displacement $(y)$ and the rotation of the rigid body $(\phi)$.

$$
y=h \cos (\phi) \Leftrightarrow \ddot{y}=-h \times \ddot{\phi} \times \sin \phi-h \times \dot{\phi}^{2} \times \cos \phi
$$

Eqs (4)-(6) can be rearranged in a system of coupled differential equations Eq (7), which relates the horizontal position of the mass centre and the angular position of the pedestrian. 


$$
\left\{\begin{array}{c}
\frac{1}{\mu} \ddot{x}-h \sin (\phi) \ddot{\phi}=h \dot{\phi}^{2} \cos (\phi)-g \\
\frac{\left[k^{2}+(\mu+1) h^{2} \sin (\phi) \cos (\phi)\right] \ddot{\phi}}{(\sin (\phi)+\mu \cos (\phi))}=\left[g h-h^{2} \dot{\phi}^{2} \cos (\phi)\right]
\end{array}\right.
$$

In these equations the horizontal component of the acceleration of the mass centre is represented by $\ddot{x}$, $k$ represents the radius of gyration of the rigid body relative to the mass centre, while $\mu$ represents the coefficient of friction. According to Wood and Simms [8], the value 0.58 for the friction coefficient can be applied, based on the work of ten different research groups. The system was solved in order to " $x$ " and " $\phi$ ", using the Runge Kutta numerical method, Fehlberg fourth-fifth order, with the initial conditions defined in Eq (8). This solution allows the calculation of the horizontal distance in the $2^{\text {nd }}$ phase " $S_{\text {din }}$ ". The simulation should be completed when the angular position " $\phi$ "reaches $90^{\circ}$.

$$
\begin{array}{lll}
t=0, & x=0 & \phi=0 \\
t=0, & \dot{x}=v_{\text {p proj }} & \dot{\phi}=0
\end{array}
$$

In the $3^{\text {rd }}$ phase of this method, the impact of the rigid body with the ground is verified for a vertical velocity given by $\mathrm{Eq}(9)$.

$$
\dot{y}_{\left(\phi=90^{\circ}\right)}=-h \dot{\phi}
$$

At the start of the $3^{\text {rd }}$ phase (vertical impact), there is a decrease in the amount of linear momentum in the horizontal direction, which results in a decrease in the horizontal velocity $v_{\text {projf }}$ with respect to $\dot{x}_{(\phi=90)}$ [9].

$$
v_{\text {projf }}=\dot{x}_{(\phi=90)}-\mu \dot{y}_{(\phi=90)}
$$

Using the principle of work and energy, the horizontal displacement in the $3^{\text {rd }}$ phase, $S_{\text {friction }}$, is determined by $\operatorname{Eq}(11)$.

$$
\frac{1}{2} M_{p} v_{p r o j f}^{2}+\left[-\mu\left(M_{p} g\right)\right] \times S_{\text {friction }}=0
$$

After this last step, it is possible to determine the pedestrian throw distance " $S_{\text {total }}$ ", being the summation of "S $S_{i m p}$ ", "S $S_{\text {din }}$ " and "S $S_{\text {friction }}$ ". Fig 2 shows the average simulated pedestrian throw distance as a function of the collision velocity, for different pedestrians and vehicles. The pedestrian throw distance increases with the collision speed. The effect of the mass of the vehicle is similar but in a very small scale. The projection distance decreases slightly with the increase of the mass of the pedestrian. The results were compared with the formulas proposed by Wood et al.[9] and with real data [4] about pedestrian throw distance derived from accident cases.

Eqs (12)-(14) represent the predicted mean values, when using the approximation formulas for the collision velocity, using approximation of Wood et al. [9], the simplified method and the advanced solution method, respectively.

$$
\begin{aligned}
& V_{\text {Col }}^{\text {mean }} \approx 9.841 \times\left(S_{\text {total }}\right)^{0.5658} \\
& V_{\text {Col }}^{\text {mean }} \approx 9.2396 \times\left(S_{\text {total }}\right)^{0.5697}
\end{aligned}
$$




$$
V_{\text {Col }}^{\text {mean }} \approx 6.264 \times\left(S_{\text {total }}\right)^{0.6573}
$$

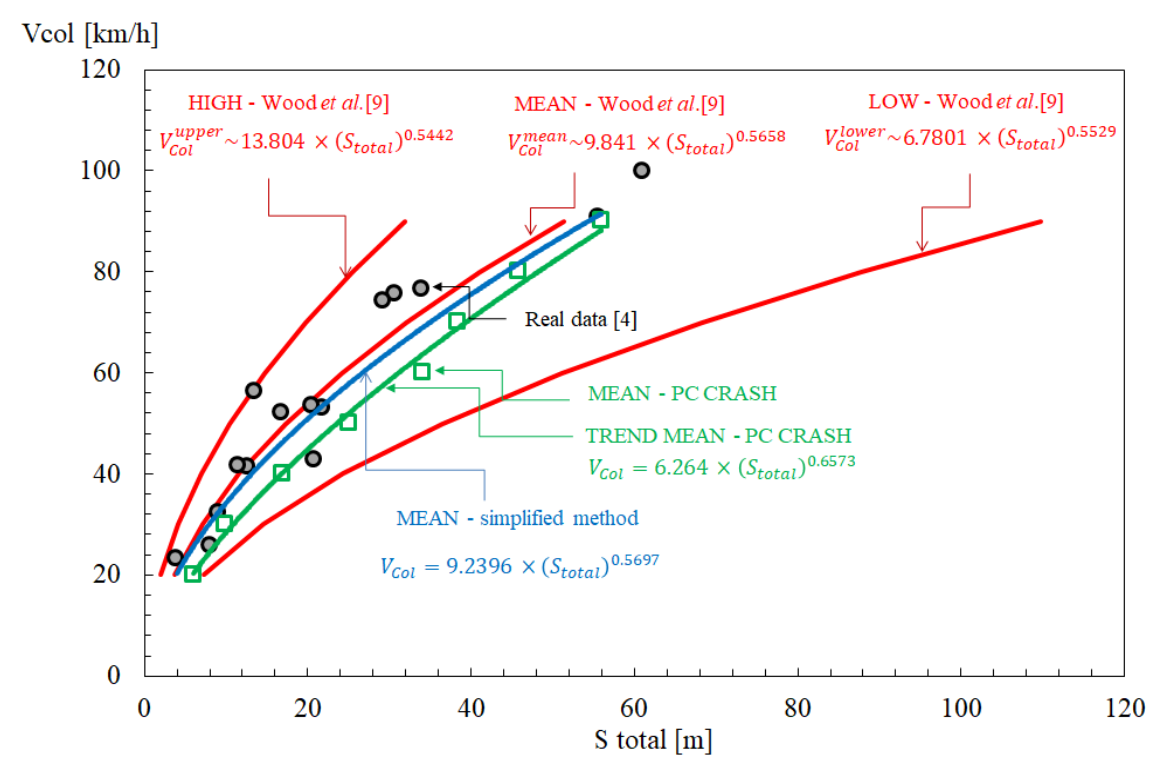

Fig.2 Pedestrian throw distance versus collision speed, for all vehicles and all pedestrians

\section{The advanced calculation method}

The pedestrian -vehicle collision 3D model was developed herein and was already validated in PC-CRASH10. This software uses a multi-body system (custom vehicle) made with 24 rigid bodies connected by 15 joints to simulate the pedestrian body motion. The vehicle model can also be subdivided in different types of vehicle masses and shapes. Three different vehicles were considered (truck tractorMAN-19.422 FLS used for Mv1, bus MAN Doppel decker ND 202 for Mv2 and bus Auwaerter - Neoplan Bus Skylinerfor Mv3, see Fig3). The collision between the vehicle (rigid body) and the pedestrian is evaluated by the calculation of the contact forces. The integration time was considered equal to 5 [ms] and the simulation time was defined up to when energy approaches zero. The total pedestrian throw distance is calculated for the pelvic region. Fig 3 represents the model for vehicle Mv3, with collision velocity Vcol1, over the pedestrian P8. The results of the 192 simulations are presented as an average of the results obtained for every pedestrian and every vehicle, see Fig 2.

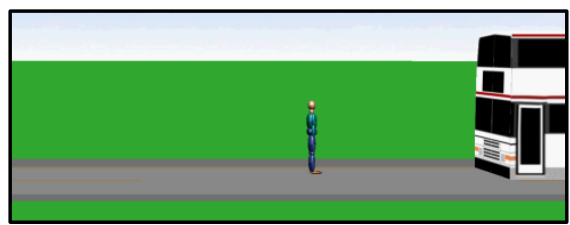

a) Pre impact position $(\mathrm{t}=-0.6[\mathrm{~s}]$, Vcol $=32.8$ $[\mathrm{km} / \mathrm{h}])$

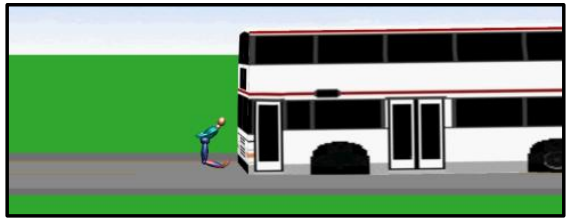

d) Genral plane motion $-2^{\text {n }} \mathrm{d}$ phase $(\mathrm{t}=0.42$ $[\mathrm{s}], \mathrm{Vcol}=11.2[\mathrm{~km} / \mathrm{h}])$

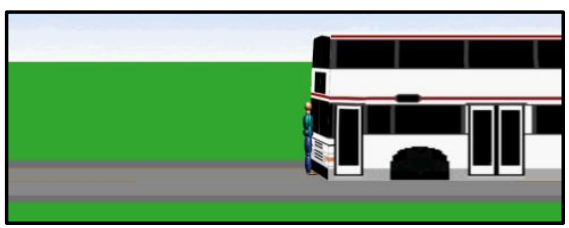

b) impact $-1^{\text {st }}$ phase $(\mathrm{t}=0[\mathrm{~s}], \mathrm{V}$ col $=20.0$ $[\mathrm{km} / \mathrm{h}])$

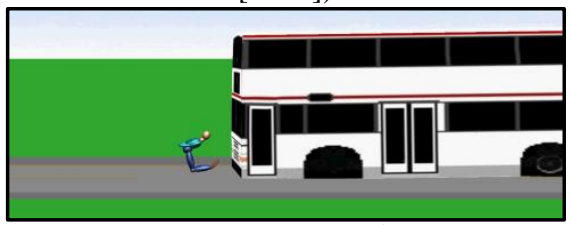

e) General plane motion $-2^{\text {nd }}$ phase $(\mathrm{t}=0.48$ $[\mathrm{s}], \mathrm{Vcol}=9.9[\mathrm{~km} / \mathrm{h}])$

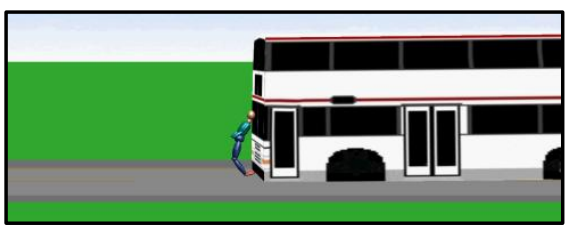

c) impact $-1^{\text {st }}$ phase $(\mathrm{t}=0.3[\mathrm{~s}], \mathrm{Vcol}=13.7$ $[\mathrm{km} / \mathrm{h}])$

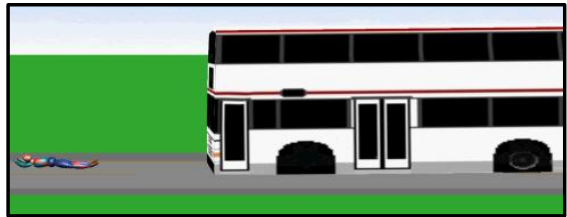

f) Friction motion $-3^{\text {rd }}$ phase $(\mathrm{t}=1.24[\mathrm{~s}]$, Vcol $=0.4[\mathrm{~km} / \mathrm{h}])$

Fig.3 Model for the collision between vehicle and pedestrian.

\section{Comparison of the solutions methods}


The prediction of the collision velocity is presented for each 15 real case, and derived from each simple formula, Eqs. (12)-(14). The relative error was also calculated for each case see Table 2.The mean average is also determined for each of the method used for the prediction of the collision velocity. The formulae of Wood et al [9] presents the smallest mean error in absolute value (9.58\% with a standard deviation of \pm 0.0875 ), while the formulae resulting from the simple calculation method presents the mean error of $10.44 \%$ with a standard deviation of \pm 0.0823 . The formulae resulting from the advance solution method presents a higher mean error $(17.76 \%$ with a standard deviation of \pm 0.1045 ). This bigger difference may be explained by the fact that the pedestrian model in PC-CRASH uses a multi-body system, consisting of several rigid bodies, to simulate the motion of the pedestrian with interaction with the vehicle and ground. No separation into the different phases of the pedestrian motion is considered in this solution method, being this model totally three-dimensional. The pedestrian model in PC-CRASH, sometimes loses the contact with the ground, reducing the friction force during the motion analysis, explaining the higher throw distance obtained.

After the $\mathrm{t}$ student test, the null hypothesis is verified. The comparison between the prediction of the collision velocity and the real data seems to be in good agreement.

Table 2 Comparison of results

\begin{tabular}{|c|c|c|c|c|c|c|c|c|c|}
\hline $\begin{array}{c}\text { Case } \\
{[4]}\end{array}$ & $\begin{array}{c}\text { Job ID } \\
{[4]}\end{array}$ & $\begin{array}{c}\mathrm{S}_{\text {total }} \\
{[4]}\end{array}$ & $\begin{array}{c}V_{\text {Col }}^{\text {Coal }} \\
{[4]} \\
\end{array}$ & $\begin{array}{c}V_{\text {Col }}^{\text {mean }} \\
{[9]}\end{array}$ & $\begin{array}{c}\text { |Error } \mid \\
{[9]} \\
\end{array}$ & $\begin{array}{c}V_{\text {Col }}^{\text {mean }} \\
{[\text { simplified] }}\end{array}$ & $\begin{array}{c}\mid \text { Error } \mid \\
\text { [simplified] }\end{array}$ & $\begin{array}{c}V_{\mathrm{Col}}^{\text {mean }} \\
{[\mathrm{PC}-\mathrm{CRASH}]}\end{array}$ & $\begin{array}{c}\mid \text { Error } \mid \\
{[\text { PC-CRASH }]}\end{array}$ \\
\hline 1 & $003^{*}$ & 61.01 & 99.97 & 100.74 & 0.01 & 96.12 & 0.04 & 93.41 & 0.07 \\
\hline 2 & $01 / 007 *$ & 55.60 & 90.68 & 95.59 & 0.05 & 91.16 & 0.01 & 87.88 & 0.03 \\
\hline 3 & $02 / 026$ & 34.04 & 76.57 & 72.42 & 0.05 & 68.93 & 0.10 & 63.65 & 0.17 \\
\hline 4 & $02 / 018$ & 30.70 & 75.64 & 68.31 & 0.10 & 64.99 & 0.14 & 59.48 & 0.21 \\
\hline 5 & $02 / 005$ & 29.20 & 74.30 & 66.40 & 0.11 & 63.17 & 0.15 & 57.55 & 0.23 \\
\hline 6 & $02 / 023$ & 21.76 & 52.99 & 56.22 & 0.06 & 53.42 & 0.01 & 47.43 & 0.10 \\
\hline 7 & 02/003 & 20.85 & 42.88 & 54.88 & 0.28 & 52.14 & 0.22 & 46.12 & 0.08 \\
\hline 8 & $02 / 009$ & 20.50 & 53.39 & 54.35 & 0.02 & 51.64 & 0.03 & 45.61 & 0.15 \\
\hline 9 & 02/031 & 16.80 & 52.02 & 48.56 & 0.07 & 46.10 & 0.11 & 40.02 & 0.23 \\
\hline 10 & 02/011 & 13.50 & 56.38 & 42.91 & 0.24 & 40.70 & 0.28 & 34.66 & 0.39 \\
\hline 11 & $00 / 006$ & 12.60 & 41.36 & 41.27 & 0.00 & 39.13 & 0.05 & 33.12 & 0.20 \\
\hline 12 & $01 / 012$ & 11.50 & 41.72 & 39.19 & 0.06 & 37.15 & 0.11 & 31.19 & 0.25 \\
\hline 13 & 01/011 & 9.00 & 32.29 & 34.12 & 0.06 & 32.31 & 0.00 & 26.55 & 0.18 \\
\hline 14 & $02 / 015$ & 8.00 & 25.67 & 31.92 & 0.24 & 30.21 & 0.18 & 24.57 & 0.04 \\
\hline \multirow[t]{3}{*}{15} & 98/001 & 3.85 & 23.22 & 21.10 & 0.09 & 19.92 & 0.14 & 15.19 & 0.35 \\
\hline & & & & Error mean & 0.0958 & & 0.1044 & & 0.1776 \\
\hline & & & & Stand. Desv. & 0.0875 & & 0.0823 & & 0.1045 \\
\hline
\end{tabular}

\section{Injury severity of pedestrians}

The GSI is a measure of the injury produced over the pedestrian head, based on the calculation of the linear acceleration for this anatomical segment (rigid body) [11]. The GSI represents an accumulated parameter over time, based on the calculation of the integral, Eq (15).

$$
G S I=\int_{t_{0}}^{t_{E}} R(t)^{2.5} d t
$$

Where $R(t)$ represents the nondimensional acceleration (ratio of the linear acceleration of the head divided by the acceleration of the gravity), $t_{0}$ represents the starting time of the simulation in seconds and $t_{E}$ the end time of the simulation. The criterion proposed by Gadd for concussion, in the case of frontal impact, is achieved when GSI equals 1000 . 
The FFC is a measure of the compression force transmitted axially on each femur of the pedestrian $F_{\text {femur }}$ [11]. The FFC injury calculation is applied to the joint force of the model. The resulting femur axial force $F_{\text {femur }}$ should be compared with the limits defined by Eq (16), in every time $t[m s]$, from $t_{0}$ up to the end of simulation $t_{E}$.

$$
\begin{aligned}
& F_{\text {feтur }}[k N] \leq 9.07-1.22 \times t \quad(t \leq 10[m s]) \\
& F_{\text {feтur }}[k N] \leq 7.85 \quad(t>10[\mathrm{~ms}]
\end{aligned}
$$

Based on these criteria, it is worth mention that the FFC criterion is normally violated when the collision velocity is higher than $60[\mathrm{~km} / \mathrm{h}]$ and the GSI criterion is normally violated when the collision velocity is higher than $40[\mathrm{~km} / \mathrm{h}]$.The variability of the human body to injury is important to be acknowledge, reason why these collision velocity values should be consider as an approximation for these limiting values.

\section{Conclusions}

In this investigation, two different methods of analysis for pedestrian overthrowing, with frontal projection were used to predict the pedestrian throw distance. These methods were compared with real data obtained for the same type of accident. The difference between the mean values of both methods is smaller than $10 \%$ for every collision velocity $\left(\mathrm{V}_{\mathrm{col}}\right)$. These methods allow establishing a relation between the pedestrian throw distance and the collision velocity of the vehicles that can be useful to the accident reconstruction for pedestrian overthrowing caused by the collision of vehicles. There is a small effect (smaller than 6\%) of the mass and height of pedestrian and mass of the vehicles on the calculation of the forward projection distance of the pedestrian. The forward projection distance is reasonably well fitted to the power law simple equation. In the case of a pedestrian overthrowing accident produced by a vehicle, the damage in the head by concussion may occur when the velocity is greater than or equal to 40 [km/h] and the damage in the femur may occur when the velocity is greater than or equal to $60[\mathrm{~km} / \mathrm{h}]$.

\section{References}

[1] National Road Safety Authority, Key indicators for national accidents (in Portuguese), Road Safety Observatory, 2011.

[2] SafetyNet, Traffic Safety Basic Facts 2008, European Road Safety Observatory, October 2008.

[3] Ravani B, Brougham D, Mason RT. Pedestrian post-impact kinematics and injury patterns. SAE Technical paper; 1981 Oct 1.

[4] James Fields, "Pedestrian/vehicle collisions collision investigation in the 21st century - Getting it Right". FCITU, west Midlands Police, Birmingham, UK, 2003.

[5] Aldman B, Thorngren L, Bunketorp O, Romanus B. An experimental model for the study of lower leg and knee injuries in car-pedestrian impacts. In International Research Council on Biomechanics of Injury conference, 1980, 180-193.

[6] Wood DP, Walsh DG. Pedestrian forward projection impact. International journal of crashworthiness. 2002 Jan 1;7(3):285-306.

[7] Lucchini E, Weissner R. Differences between the kinematics and loadings of impacted adults and children; Results from dummy tests. InProc 1980 International Research Council on the Biomechanics of Impact Conf 1980.

[8] Wood DP, Simms CK. Coefficient of friction in pedestrian throw. Impact, Spring. 2000:12-4.

[9] Wood DP, Simms CK, Walsh DG. Vehicle-pedestrian collisions: Validated models for pedestrian impact and projection. Proceedings of the Institution of Mechanical Engineers, Part D: Journal of Automobile Engineering. 2005 Feb 1;219(2):183-95.

[10] Moser A, Steffan H, Kasanicky G. The pedestrian model in PC-crash: The introduction of a multibody system and its validation. SAE transactions. 1999;108(6):794-802.

[11] Tnomadymo BY, MADYMO Theory Manual - version 6.2, June 2004. 